\title{
Mini-Review: Sarcomatoid Non-Clear Cell Renal Cancer
}

\author{
Sai Gadde ${ }^{1,2}$, Benjamin Jones ${ }^{1} \&$ Christopher Old ${ }^{1,2}$ \\ ${ }^{1}$ Grandview Medical Center, Birmingham, Alabama, USA \\ ${ }^{2}$ Brookwood Baptist Health System, Birmingham, Alabama, USA \\ Correspondence: Sai Gadde, Grandview Medical Center, Birmingham, Alabama, USA. E-mail: \\ sai.gadde@bhsala.com
}

$\begin{array}{lc}\text { Received: March 29, } 2017 & \text { Accepted: April 28, } 2017 \quad \text { Online Published: July 8, } 2017 \\ \text { doi:10.5539/cco.v6n2p1 } & \text { URL: http://dx.doi.org/10.5539/cco.v6n2p1 }\end{array}$

\begin{abstract}
Sarcomatoid differentiation can occur within any subdivision of renal cell carcinoma. Although the prevalence of sarcomatoid renal cell cancer has been reported to be anywhere from 1-15\% of renal cancers, sarcomatoid non-clear cell renal carcinoma is actually very rare. Studies on these cancers are generally limited to tens of patients. One reason for the paucity of patients with non-clear cell sarcomatoid renal cell cancer is the aggressive nature of this disease. We present a case of sarcomatoid non-clear cell renal carcinoma and review of the treatment for these difficult cases based on the available literature.
\end{abstract}

Keywords: clear cell, non-clear cell, surgery, chemotherapy, immunotherapy

\section{Introduction}

Renal cell carcinoma is one of the ten most prevalent cancers in both men and women in the United States. Ninety percent of kidney cancers arise from the renal parenchyma (within the proximal tubules of the kidney), and are therefore called renal cell cancers (RCC) (http://emedicine.medscape.com/article/1612043-overview\#a2). RCC is histologically divided into two main groups: clear cell renal cancer and non-clear cell renal cancer (which is made of many subdivisions). Clear cell carcinomas are much more common (about $70 \%$ of renal cell cancers) and they have a pale appearance to the cell with clear cytoplasm on microscopy (https://old.cancer.org/acs/groups/cid/documents/webcontent/003107-pdf.pdf). Sarcomatoid differentiation in RCC refers to tumors with malignant, spindle-shaped histology. It is not a distinct histologic diagnosis, but can occur anywhere along the spectrum of renal cancer.

Sarcomatoid renal cell cancers have a poor overall prognosis. Treatment outcomes in metastatic clear cell carcinoma have been extrapolated to the treatment of less common RCC subtypes. However, there have been further attempts within the last two decades to evaluate small groups of patients with non-clear cell renal carcinoma. Most of the initial studies on these cancers were retrospective analyses, but there have been some recent prospective trials of non-clear cell RCC. Finally, as in other areas of oncology, the role of immunotherapy is being explored in all subtypes of RCC.

\section{Description of Case Report}

A 56 year old Caucasian male with no past medical history presented with dyspnea, which had been refractory to over the counter expectorants. The patient denied any B-symptoms and didn't have any GI or weight loss symptoms on presentation. The patient has no known family history of any cancers. He had not undergone preventive health screenings such as colonoscopy and PSA evaluation in the past as he has not utilized medical care in the previous twenty years.

He was noted to initially have a large left sided pleural effusion on chest X-ray; this effusion was causing near complete collapse of left lung with mediastinal shift. A subsequent CT chest revealed a significant amount of atelectasis and a loculated effusion. Cardiothoracic surgery was then consulted to assist with the management of this located effusion, and intraoperative management led to the finding of a spindle cell neoplasm that involved pleura and left lower lobe. While recovering from thoracotomy, he had a CT of his abdomen/pelvis which revealed a right sided $6 \mathrm{~cm}$ kidney mass consistent with renal cell carcinoma. His baseline creatinine $(\mathrm{Cr})$ was unknown due to limited previous medical care but his $\mathrm{Cr}$ when he presented to the hospital was 1.32 . He also had a mild anemia with a hematocrit of 34 , but there were no significant other lab values. 


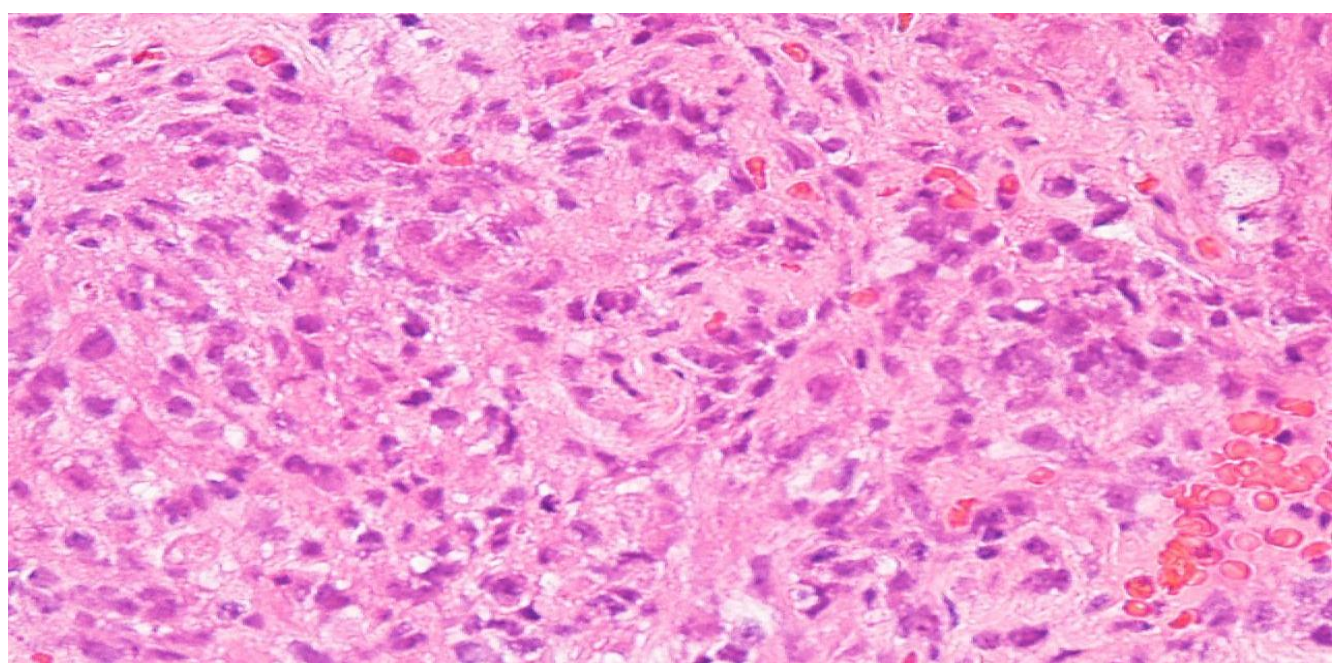

Figure 1. Histology from Above Case - Atypical Epithelial and Spindle Cells from Metastatic Lung Tissue

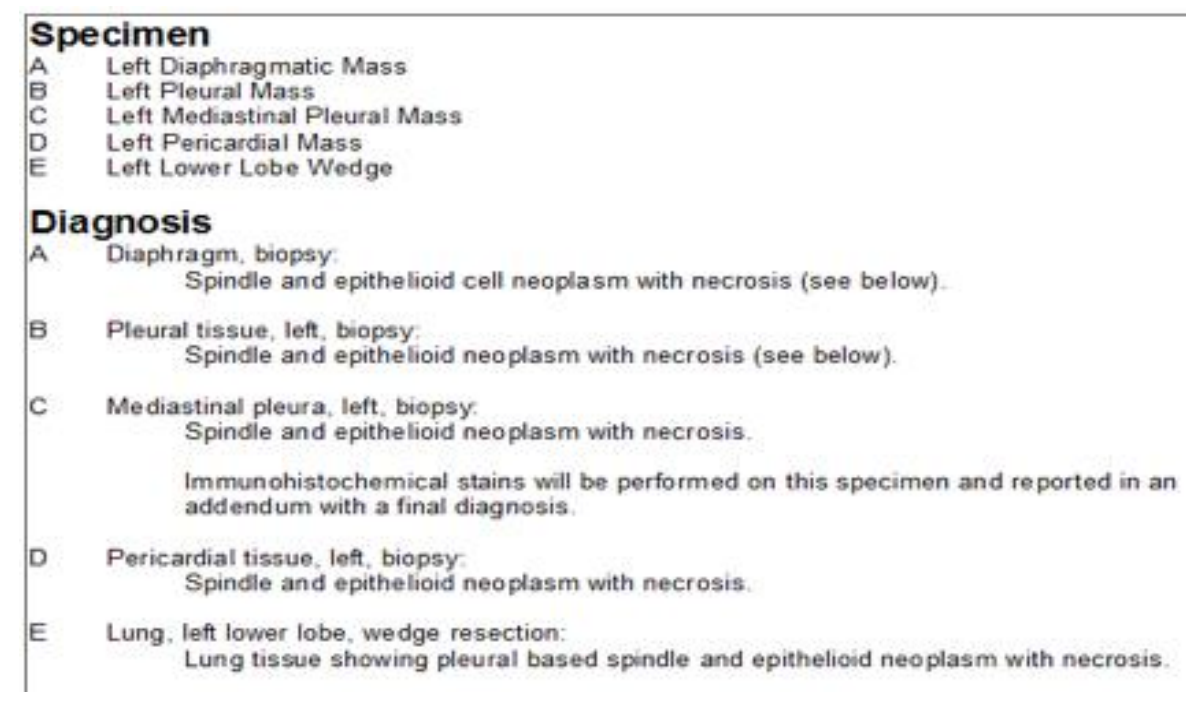

- C Mediastinal pleura, left, biopsy:

- The lesional cells have the following immunohistochemical staining pattern:

$\begin{array}{lll}\text { - } & \text { Pancytokeratin } & \text { - focally positive, } \\ \text { - } & \text { Cytokeratin 7 } & \text { - negative, } \\ \text { : } & \text { Cytokeratin } 20 & \text { - negative, } \\ \text { - } & \text { Calretinin } & \text { - negative, } \\ \text { D2-40 } & \text { - negative, } \\ \text { : } & \text { BerEp4 } & \text { - negative, } \\ \text { - } & \text { WT-1 } & \text { - negative, } \\ \text { HBME-1 } & \text { - negative, } \\ \text { - } & \text { S100 } & \text { - negative, } \\ \text { - } & \text { Mart-1 } & \text { - negative, } \\ \text { - } & \text { SMA } & \text { - positive, } \\ \text { PAX-8 } & \text { RCC } & \text { - focal positive, } \\ \end{array}$

In summary, the immunohistochemical staining pattern is not entirely specific; however, given the recent C广 finding of â a large renal mass, the possibility that these foci identified in association with the pleura are metastatic from a sarcomatoid renal cell carcinoma or renal cell carcinoma with sarcomatoid features cannot be totally excluded.

Figures 2\&3. Pathology reports revealing spindle cell histology and likely Sarcomatoid Renal Cell Cancer 


\section{Discussion}

Clear cell carcinoma traditionally describes kidney tumors with clear cells equal to or more than $50 \%$ of the specimen. Tumors with less than 50\% clear cells are called non-clear cell carcinoma (Xu \& Wu, 2017). The sarcomatoid differentiation of renal cell cancer (sRCC) can occur in any renal cell carcinoma subtype, including clear cell carcinoma and non-clear cell carcinoma. A cut off of 30\% sarcomatoid features in primary tumor is commonly used to classify a tumor with sarcomatoid histology.

On immunohistochemical staining, sarcomatoid tumors stain for cytokeratin AE1/ AE3 in 97\% of cases and vimentin in 56\% of cases. Necrosis is present in $90 \%$ and microvascular invasion in $30 \%$ of cases, both of which are considered high risk features. Sarcomatoid cancers have a higher proliferative rate than other renal tumors based on Ki-67 staining. These tumors are thought to have greater p53 mutation frequency. An analysis of fourteen sarcomatoid tissue specimens completed by Oda et al revealed 11/14 with mutations versus adjacent cancerous tissue, which had 2/14 with p53 mutations. However, there has been debate regarding this finding as adjacent tissue to sarcomatoid cancer also had low p53 expression (Shuch, Bratslavsky, Linehan \& Srinivasan, 2012).

Generally, sarcomatoid renal tumors present with a large mean tumor size of 9-10 cm, and ninety percent of cases produce symptoms at presentation. These symptoms can potentially include hematuria, anemia, back pain or mass on flank, fatigue, weight loss, and appetite changes. It has been noted that $45-84 \%$ of these patients will have metastases at time of presentation as well. Core biopsies are necessary as FNA does not reliably allow for acquisition of adequate tissue. A study completed at MD Anderson revealed that only $10 \%$ of sRCC patients who had nephrectomy had histology demonstrating the same components on preoperative renal biopsy (Brookman-May et. al., 2013). The general prognosis for renal cell cancer with sarcomatoid differentiation is only about 4-9 months of median survival due to the aggressive nature of the disease.

\subsection{Surgical Treatment of Renal Cancer}

Nephrectomy is the treatment of choice for most small localized tumors, with larger bulky tumors requiring radical nephrectomy. Given the aggressive biology of sRCC, physicians have questioned the benefit of cytoreductive surgery in the metastatic setting. Previous experience in these patients showed that $60 \%$ of sRCC patients could not have systemic therapy after surgery due to rapid disease progression. The patients' functional status after these surgeries did not improve enough to be able to tolerate chemotherapy.

Another research study focused primarily on sarcomatoid renal cell carcinoma (sRCC) with specifically localized and non-metastatic disease (Merrill et al., 2017). Median time to recurrence in groups with nephrectomy (56 out of 77 patients) was 26.2 months (Merrill et al., 2017). Most of the patients (75/77) had radical nephrectomy over partial nephrectomy; a subgroup (27 pts) also had undergone IVC thrombectomy. There was no systemic treatment used in these patients as part of initial therapy. Metastasis, nodes, and lymphovascular invasion during the follow-up were shown to be factors that negatively impacted overall survival. The most common sites of initial recurrence from local disease were lung (45\%), bone, and liver. About $71 \%$ of the patients in this trial received systemic treatment and $29 \%$ received radiation treatment after the first recurrence; $36 \%$ also had metastectomy (Merrill et al., 2017).

\subsection{Targets of Targeted Treatment}

Systemic therapy is evolving for advanced RCC, with most novel therapies being studied in patients with clear cell RCC. The majority of current treatment for non-clear cell renal cancer treatment has focused on tyrosine kinase inhibitors (TKIs) or mTor inhibitors (mammalian target of Rapamycin). There has additionally been some recent attention towards immunotherapy, such as PD-1 (programmed death 1) inhibitors as these group of drugs have demonstrated strong results in cancers such as melanoma, non-small cell lung cancer, and a few other types of cancer.

One of the main targets for drug therapy is the upregulation of vascular endothelial growth factor (VEGFR) activity in multiple renal cancers that has corresponded to the uncontrolled modulation of the hypoxic response. This has been demonstrated by higher levels of hypoxia inducible factor I (HIF 1) expression in renal carcinoma patients. Multiple agents (e.g., sorafenib, sunitinib, bevacizumab, pazopanib, axitinib, temsirolimus, everolimus) have been identified as abrogators of VEGF mediated signaling (Sankin, Hakimi, Hsieh, \& Molina, 2015).

An oral angiogenesis inhibitor, pazopanib, targeting VEGFR (vascular endothelial growth factor receptor), PDGFR (platelet-derived growth factor receptor), and the c-kit tyrosine kinase demonstrated significant improvements in progression-free survival and tumor response in both treatment-naive and pre-treated patients with metastatic renal cell carcinoma. Paralleling pazopanib's effects, sorafenib (an oral multikinase inhibitor of 
VEGF receptors, platelet-derived growth factor receptors, and Raf kinases) was found in a phase III randomized control trial to lead to significantly longer median progression-free survival in metastatic renal cell cancer patients compared to placebo. In 2007, sunitinib, another orally administered inhibitor of tyrosine kinases ranging from VEGFRs to PDGFRs, was found to contribute to significantly higher progression-free survival and response rate in metastatic renal cell carcinoma (mRCC) patients participating in a phase III trial. Pazopanib, sunitinib, and axitinib had significantly better outcomes for metastatic renal cell carcinoma patients (Tsimafeyeu, 2017).

Given that a number of downstream mTOR (mammalian target of rapamycin) effectors regulating angiogenesis, metabolism, and cell growth have been found to be deregulated in cancers, various targeted therapies such as temsirolimus and everolimus have been developed to hinder mTOR signaling. Everolimus does not target mTOR2, which is thought to have a significant role in tumor proliferation. Temsirolimus is an mTor kinase inhibitor which binds to intracellular protein, FKBP-12 and forms a complex that inhibits kinase activity of mTOR), leading to cell cycle arrest.

\subsection{Targeted Treatment, Studies in Non-Clear Cell Renal Carcinoma}

Many studies have focused on the broad overall category of non-clear cell renal carcinoma. In a randomized phase 2 trial of non-clear cell RCC, the ESPN trial, researchers sought to evaluate the assumption that mTOR (mammalian target of rapamycin) inhibitors like everolimus may benefit "poor-risk" disease patients such as those with non-clear cell renal carcinoma (nccRCC) in terms of improved progression-free survival (PFS) and overall survival (OS). Employing a crossover study design, researchers evaluated the premise that median PFS would be improved from 12 weeks with sunitinib to 20 weeks with everolimus. Through evaluation of a sample of 68 participants (27 papillary, 11 chromophobe, 9 unclassified, 7 translocated, 13 sarcomatoid, 1 oncocytic), researchers noted significantly higher overall survival in the sunitinib arm (not reached vs 10.5 months, $p=0.01$ ). This study was terminated early due to this finding.

Gore et al. enrolled approximately 4500 patients in a trial (12\% with non-clear cell renal cancer) of sunitinib (a tyrosine kinase inhibitor). A group of patients were treated previously with cytokines and other cancer therapy before taking part in this trial, which would be a limitation to the study. Response to treatment was noted in $8 \%$ of patients with nccRC but disease control was seen in $51 \%$ of patients. Median progression free survival (6.0 vs. $9.4 \mathrm{mo})$ and overall survival (12.2 vs. 18.7 months) was less in non-clear cell renal carcinoma patients in comparison to the overall study group. Another trial evaluated 53 patients with papillary or chromophobe, non-clear cell metastatic RCC and sunitinib had greater progression free survival compared to sorafenib, also a tyrosine kinase inhibitor (11.9 months vs. 5.1 months, respectively). Twenty percent of sunitinib treated patients survived 5 years or more in one of trials with non-clear cell renal carcinoma patients (Xu \& Wu, 2017).

A phase III trial, ARCC, compared temsirolimus alone with temsirolimus and IFN-alpha combined in non-renal cell carcinoma patients. Temsirolimus monotherapy had improved overall survival (10.3 vs. 7.3 months, $\mathrm{p}=$ 0.008) and progression free survival (5.5 mo vs. 3.1 months) (Bellmunt \& Dutcher, 2013). According to this trial which revealed the benefit of temsirolimus as a first line drug, certain criteria were used to predict poor-prognosis and optimal benefit of this drug in the nccRCC setting. These criteria included 3 or more criteria of the following: high LDH level, high serum calcium, low hemoglobin, time from diagnosis to need for systemic treatment $<1$ year, lower Karnofsky performance status, and presence of metastatic lesions.

The National Comprehensive Cancer Network (NCCN) gives sorafenib or sunitinib gives category 2 A ratings for the treatment of sarcomatoid RCC and temsirolimus a category I rating in poor prognostic groups, such as metastatic non-clear cell RCC.

\subsection{Chemotherapy in Sarcomatoid Renal Cell Cancer}

A few other studies involved subsets of patients with sarcomatoid renal cell cancer and used different modalities of systemic chemotherapy. One study, by Nanus et al., completed in a small group of patients $(\mathrm{n}=10)$ revealed survival of up to 6 and 8 years when gemcitabine (cytosine analogue) and doxorubicin (anthracycline) combination treatments were used. A phase II study, ECOG (Eastern Cooperative Oncology Group) - 8803 followed this study to assess the role of this drug therapy in 38 patients. The median progression free survival was noted to be 3.5 months and overall survival was at 8.8 months. Combination of gemcitabine and doxorubicin had response rate of $15.8 \%$. The Dana Farber cancer institute was working on a Phase II trial with sunitinib and gemcitabine (sunitinib given in first two weeks and gemcitabine on days 1 and 8), which had demonstrated a poor median time to progression of 4.6 months.

Some of these chemotherapy treatments have received a category 3 rating (gemcitabine + doxorubicin or 
gemcitabine + capecitabine).

Table 1. Renal Cell Carcinoma Treatment Guidelines

\begin{tabular}{|c|c|c|}
\hline \multicolumn{3}{|c|}{ Renal Cell Carcinoma Guidelines Summary } \\
\hline & Treatment & Options \\
\hline Types/Stages of Cancer & Category I/ PreferredRecommendations & $\begin{array}{lllll}\text { Category II } & \text { Recommendations/ } & 2^{\text {nd }} & \text { Line } \\
\text { Therapy } & & & & \\
\end{array}$ \\
\hline $\begin{array}{l}\text { Clear/ Non-Clear Cell } \\
\text { Renal Cancer: Stage I } \\
\text { (pT1a) }\end{array}$ & Partial Nephrectomy & $\begin{array}{l}\text { Radical Nephrectomy (if partial not possible or } \\
\text { tumor in central location) }\end{array}$ \\
\hline $\begin{array}{l}\text { Clear/ Non-Clear Cell } \\
\text { Renal Cancer: Stage Ib, } \\
\text { II, III }\end{array}$ & Radical or Partial Nephrectomy & Radical or Partial Nephrectomy \\
\hline $\begin{array}{l}\text { Clear Cell Renal Cancer: } \\
\text { Stage IV }\end{array}$ & $\begin{array}{l}\text { Surgical: Nephrectomy +/- Metastectomy } \\
\text { ChemoTx: Clinical Trial; Pazopanib, Sunitinib, } \\
\text { Bevacizumab + IFN (All Cat 1); Temsirolimus (Cat } 1 \\
\text { in poor prognosis pts); Axitinib }\end{array}$ & $\begin{array}{l}\text { ChemoTx: High dose I12, Sorafenib; Bevacizumab, } \\
\text { Cabozantinib, Nivolumab, Axitinib, Lenvatinib + } \\
\text { Everolimus; Everolimus, }\end{array}$ \\
\hline $\begin{array}{l}\text { Non-Clear Cell Renal } \\
\text { Cancer: Stage IV }\end{array}$ & $\begin{array}{l}\text { Temsirolimus (Cat I); Clinical Trial, Sunitinib (both } \\
\text { also preferred) }\end{array}$ & $\begin{array}{l}\text { Axitinib, Bevacizumab, Cabozantinib, Erlotinib, } \\
\text { Everolimus, Lenvatinib }+ \text { Everiloimus, } \\
\text { Nivolumab, Pazopanib, Sorafenib, Best supportive } \\
\text { care }\end{array}$ \\
\hline
\end{tabular}

\footnotetext{
* Table based on summary of National Comprehensive Cancer Network (2016) v 2.2017 Renal Cell Carcinoma Guidelines

* Cat I, II = Category I and Category II recommendations, respectively

* Primary division of this table is Category I and II recommendations per their guidelines

* Some of the 1 st line and $2^{\text {nd }}$ line treatment overlapped for Stage IV clear cell cancer and were excluded from being listed again as $2^{\text {nd }}$ line tx if they were already listed as $1^{\text {st }}$ line treatment
}

\subsection{Immunotherapy in Renal Cell Carcinoma}

Immunotherapies work by enhancing immune recognition of malignant cells. Programmed death-1 (PD-1) is a surface glycoprotein on T cells, B Cells, and macrophages that inhibits the activation of these cells. There was a phase I study completed with nivolumab $(1 \mathrm{mg} / \mathrm{kg})$ and ipilimumab $(3 \mathrm{mg} / \mathrm{kg})$, which showed almost a $48 \%$ response rate in clear cell renal carcinoma when both medications were combined; the median survival for both therapies used together was 38.3 weeks. There is however, limited biomarker data though to visualize efficacy but nonspecific chemokine such as CXCL9 and CXCL10 had been noted to be correlated with potential efficacy of drug. So, nivolumab has been approved for the treatment of metastatic clear cell renal cancer due to its tolerance profile in comparison to ipilimumab. However, in the case of non-clear cell renal cancer, there is a phase $3 \mathrm{~b} / 4$ safety trial that is ongoing at this time.

\section{Conclusion}

Our patient with no significant past social, family, or medical history was incidentally noted to have sarcomatoid non-clear cell renal carcinoma after initial presentation of dyspnea which led to the detection of likely pulmonary metastases. Due to the paucity of cases of sRCC, the treatment has been extrapolated from trials involving patients with the broader category of non-clear cell renal carcinoma. The current NCCN guidelines give a respective category of I and category IIA ratings to temsirolimus, sunitinib, or a clinical trial for the treatment of non-clear cell carcinoma. In addition, there are new trials ongoing which are attempting to reassess the role of effective clear cell renal carcinoma drugs for use in non-clear cell carcinoma, such as the ongoing pazopanib trial. In our patient's case, the potentially upcoming treatment modalities are being attempted with pazopanib as his first treatment drug, and there are plans to potentially try nivolumab if disease progression were to occur. The potential role of immunotherapy in non-clear cell or sarcomatoid renal cell cancer monitored over time and its effect when combined with chemotherapy will be interesting future studies to follow.

\section{References}

Bellmunt, J., \& J. Dutcher. (2013), Targeted therapies and the treatment of non-clear cell renal cell carcinoma. Annals of Oncology, 24(7), 1730-740. https://doi.org/10.1093/annonc/mdt152

Brookman-May, Sabine et al. (2013). Prognostic Effect of Sarcomatoid Dedifferentiation in Patients With Surgically Treated Renal Cell Carcinoma: A Matched-Pair Analysis. Clinical Genitourinary Cancer, 11(4), 
465-70. http://dx.doi.org/10.1016/j.clgc.2013.04.026

Kidney Cancer - Renal Cell Carcinoma. (2014). American Cancer Society. Retrieved January 17, 2017, from https://old.cancer.org/acs/groups/cid/documents/webcontent/003107-pdf.pdf

Merrill, Megan M et al. (2015). Clinically nonmetastatic renal cell carcinoma with sarcomatoid dedifferentiation: Natural history and outcomes after surgical resection with curative intent. Urologic Oncology: Seminars and Original Investigations, 33(40), 1-22. http://dx.doi.org/10.1016/j.urolonc.2014.11.021

Kidney Cancer. (2017). NCCN Clinical Practice Guidelines in Oncology. National Comprehensive Cancer Network, 2, 1-55.

Pathology of Clear Cell Renal Cell Carcinoma. (2016). Medscape. Retrieved Jan 17, 2017, from http://emedicine.medscape.com/article/1612043-overview\#a2

Sankin, Alexander, A. Ari Hakimi, James J. Hsieh, \& Ana, M. Molina. (2015). Metastatic Non-Clear Cell Renal Cell Carcinoma: An Evidence Based Review of Current Treatment Strategies. Frontiers in Oncology, 5, 1-7. https://doi.org/10.3389/fonc.2015.00067

Shuch, B., Bratslavsky, G., Linehan, W. M., \& Srinivasan, R. (2012). Sarcomatoid Renal Cell Carcinoma: A Comprehensive Review of the Biology and Current Treatment Strategies. The Oncologist, 17(1), 46-54. https://doi.org/10.1634/theoncologist.2011-0227

Tsimafeyeu, Illya. (2017). Management of non-clear cell renal cell carcinoma: Current approaches. Urologic Oncology: Seminars and Original Investigations, 5-13. http://dx.doi.org/10.1016/j.urolonc.2016.07.011

Xu, Kevin Y., \& Shenhong, Wu. (2015). Update on the treatment of metastatic clear cell and non-clear cell renal cell carcinoma. Biomarker Research, 3(1), 1-5. https://doi.org/10.1186/s40364-015-0030-7

\section{Copyrights}

Copyright for this article is retained by the author(s), with first publication rights granted to the journal.

This is an open-access article distributed under the terms and conditions of the Creative Commons Attribution license (http://creativecommons.org/licenses/by/4.0/). 